BULL. AUSTRAL. MATH. SOC.

VOL. 7 (1972), 429-436.

\title{
Maximal perfect spaces
}

\section{Ivan Baggs}

Let $(X, T)$ be a topological space (we assume $T_{1}$ throughout) where every point is a limit point. The purpose of this note is to present an internal construction of a maximal perfect topology on $(X, T)$. The existence of a maximal connected Hausdorff space has not been demonstrated. However, this construction of a maximal perfect topology is useful in constructing connected Hausdorff spaces which cannot be embedded in a maximal connected Hausdorff space.

Let $(X, T)$ be a topological space in which every point is a limit point, then $(X, T)$ is said to be perfect. (Throughout this note, all spaces are assumed to be $T_{1}$.) A topological space $(X, T)$ is maximal perfect if $(X, T)$ is perfect and for every topology $T^{\prime} \supsetneqq T,\left(X, T^{\prime}\right)$ is not perfect. It follows from an application of Zorn's Lemma that, if $(X, T)$ is a perfect topological space, there exists a topology $T^{\prime} \supset T^{\prime}$ such that $\left(X, T^{\prime}\right)$ is maximal perfect. The main aim of this note is to present an internal construction of a maximal perfect topology for any given perfect topological space. The advantage of this construction arises when one wishes to know which sets are open in a maximal perfect topology. This maximal perfect topology is constructed by using $N$-sets (see Definition 1$)$ and a particular filter, $F$, of dense subsets of $(X, T)$. It is also shown that, if $\sigma \supset T \vee F$ and $(X, \sigma)$ is perfect, then, if $G \in T \vee F, G=F \cap M$, where $F \in F$ and $M$ is an $N$-set.

The problem of the existence of a maximal perfect topology arose

Received $6 \mathrm{July}$ 1972. This research was partly supported by a grant from the INational Research Council of Canada. The author is deeply indebted to Professor Lee Mohler for many stimulating discussions. 
naturally from the consideration of the existence of a maximal connected Hausdorff space. A connected Hausdorff space $(X, T)$ is maximal cunnected if for every topology $T^{\prime} \supsetneqq T,\left(X, T^{\prime}\right)$ is not connected (see [2]). The author used the type of particular construction of a maximal perfect topology, as presented in this note, to construct an example of a countable connected Hausdorff space $(X, T)$ which is not first countable at any point and such $T$ is not contained in any maximal connected topology on $X \quad[1]$.

If $(X, T)$ is a topological space, then we will say a set $K \subset X$ is T-dense in $X$ if $X$ is contained in the closure of $K$ in the $T$ topology.

Also, $K$ is $T$-open if $K \in T$, and $K^{c}$ will be used to denote the complement of $K$.

For the remainder of this note, we will assume that $(X, T)$ is an arbitrary but fixed perfect topological space. Let

$$
H=\left\{F \subset X \mid F^{C} \text { is nowhere dense in } X\right\} \text {. }
$$

Then, $B$ is a filtex of dense subsets of $X$. It follows from Zorn's Lemma that there exists a maximal filter $F$, containing $B$, such that for every $F \in F, F$ is a dense subset of $X$. Throughout, we will use $F$ to denote this maximal filter on $X$.

DEFINITION 1. A set $G \subset X$ is an $N$-set if for every $x \in G$ and every open set $U \in T$ and containing $x, U \cap G$ contains a $T$-open set.

It follows, immediately, from the definition, that if $U \in T$, then $U$ is an $N$-set. However, there may exist $N$-sets which are not $T$-open; for example, if $G$ is the subset of the real line defined as follows:

$$
G=\{0\} \cup\left\{\left(\frac{1}{2 n}, \frac{1}{2 n-1}\right) \mid n=1,2, \ldots\right\} \text {. }
$$

DEFINITION 2. A collection of $N$-sets, $G$, is an $N$-family if $G$ is closed under finite intersection.

If $G_{1}$ and $G_{2}$ are two $N$-families, we will say that $G_{1} \leq G_{2}$ if for every $G \in G_{1}, G \in G_{2}$. It follows from Zorn's Lemma that given any 
$N$-family $G$, there exists a maximal $N$-family $M$, such that $G \leq M$. In what follows, $M$ will be used to denote a maximal $N$-family on $X$.

If $A$ and $B$ are two families of subsets of $X$, by $A \vee B$ is meant the topology which is generated by the subbase

$$
P=\{C \mid C \in A \text { or } C \in B\} \text {. }
$$

LEMMA 1. Let $T^{\prime}=F \vee M$; then $T^{\prime} \supset T$ and $\left(X, T^{\prime}\right)$ is perfect.

Proof. Since $T \subset M$, it follows that $T \subset T^{\prime}$. Suppose $\left(X, T^{\prime}\right)$ is not perfect. This implies that there exists some $x \in X$ such that $\{x\} \in T \vee F$. Therefore, $\{x\}=F \cap G$, where $F \in F$ and $G \in M$. However, since $G$ contains a non-empty $T$-open set $U$ and $F \cap U$ is T-dense in $U$, it follows that $F \cap G \neq\{x\} ;$ a contradiction. So, $\left(X, T^{\prime}\right)$ is perfect.

Henceforth, $T^{\prime}$ will be used to denote the topology $F \vee M$. Let us assume there exists a topology $T^{*}$ on $X$, such that $\left(X, T^{*}\right)$ is perfect and $T^{*} \supsetneqq T^{\prime}$.

LEMMA 2. If $P \in T^{*}, P \subset U$, where $U \in T$, and $P$ is $T$-dense in $U$, then $P \in T \vee F$.

Proof. Suppose $P \subset U$ and $P$ is $T$-dense in $U \in T$ and $P \notin T \vee F$. If, for each $F \in F, P \cap F$ is $T$-dense in $U$, then $F \cap\left(P \cup \mathscr{V}^{f}\right)$ is dense in $X$ for all $F \in F$. So, since $F$ is maximal, this would inply that $P \cup U^{c} \in F$. However, if $P \cup U^{f} \in F$, then $P=\left(P \cup U^{\mathcal{C}}\right) \cap U$ and $P \in T \vee F$. If, on the other hand, there exists some $F \in F$ such that $F \cap P$ is not $T$-dense in $U$, then there exists some T-open set $V \subset U$ such that $V \cap(F \cap P)=\varnothing$. Let $x \in V$ such that $x \in P$ and $x \neq F$. Put $F_{1}=F \cup\{x\}$. Then $F_{1} \in F$ and is, therefore, open in $T \vee F$. This would imply that $F_{1} \cap(P \cap V)=\{x\}$ and since $F_{1} \cap P \in T^{*}$, this implies $\{x\}$ is open in $T^{*}$. However, since $T^{*}$ is perfect, this is impossible. Therefore, for each $F \in F, F \cap P$ is T-dense in $U$, and so $P \in T \vee F$.

COROLLARY. If $P \in T^{*}, P \subset U$, where $U \in T$ and $P$ is $T$-dense in $U$, then $P \in T^{\prime}$. 
DEFINITION 3. Let $P \in T^{*} . P$ has property $\beta$ if for every $x \in P$ there exists a $T^{*}$-open set $U_{x} \subset P$ and containing $x$ such that $U_{x}=\left(\bigcup_{\alpha \in A} K_{\alpha}\right) \cup L$, where

(a) for each $\alpha \in A, K_{\alpha} \subset U_{\alpha} \in T$ and $K_{\alpha}$ is $T$-dense in $U_{\alpha}$,

(b) no subset of $L$ is $T$-dense in any $T$-open set, and

(c) if $x \in L$, then $x \notin K_{\alpha}$, for any $\alpha$.

LEMMA 3. Let $P$ be a set with property $B$ such that $P \in T^{*}$. Let $x \in P$ and $U_{x}=\left(\cup_{\alpha \in A} K_{\alpha}\right) \cup L$ be as in Definition 3. Then, for every $y \in L \cap U_{x}$ and for every $T^{\prime}$-open set $U$ containing $x, U \cap U_{\alpha} \neq \emptyset$, for some $\alpha \in A$.

Proof. Suppose there exists some $x \in P$ and some $y \in L \cap U_{x}$ and a $T^{\prime}$-open set $U$ containing $y$ such that $U \cap U_{\alpha}=\varnothing$, for all $\alpha \in A$. We may assume without loss of generality that $U=F \cap(\operatorname{InM})$, where $F \in F$, $I \in T$ and $M \in M$. Since $M$ is an $N$-set, $M \cap I$ contains a non-empty $T$-open set. Let $Q$ be any non-empty $T$-open set contained in $I \cap M$. Then, $Q \cap U_{\alpha}=\emptyset$, for all $\alpha \in A$; for, if there exists some $\alpha \in A$, such that $Q \cap U_{\alpha} \neq \emptyset$, and since $Q \subset(I \cap M)$, this implies that $U \cap U_{\alpha} \neq \emptyset$. Also $(I \cap M) \cap K_{\alpha}=\emptyset$, for all $\alpha \in A$. If there exists an $x \in(I \cap M) \cap K_{\alpha}$, for some $\alpha \in A$, then, since $K_{\alpha} \subset U_{\alpha}$ and $I \cap M$ is an $N$-set, if $U^{\prime}$ is an $T$-open set containing $x, U^{\prime} \cap(I \cap M) \cap U_{\alpha}$ would contain a T-open set. This would contradict the fact that $U_{\alpha} \cap Q=\emptyset$, for each open set $Q \subset(I \cap M)$. Therefore, $(I \cap M) \cap k_{\alpha}=\varnothing$, for all $\alpha \in A$.

$y \in U \cap U_{x}$ and $T^{*}$ is perfect, so there exists some $p \in U \cap U_{x}$ and $p \neq y$. Since $U \cap U_{\alpha}=\varnothing$, for al $\alpha$ and $k_{\alpha} \subset U_{\alpha}, p \notin K_{\alpha}$, for any $\alpha \in A$. Therefore, $p \in L$. Let $V$ be any $T$-open set containing $p$. Put $H=V \cap(I \cap M)$. Since $p \in U$ and $U=F \cap(I \cap M)$, it follows that 
$p \in E$. Also, since $(I \cap M) \cap K_{\alpha}=\emptyset$, for all $\alpha \in A, L \supset B \cap P$. From the definition of $L$, it follows that $H \cap P$ is nowhere dense in $(X, T)$. So, $(H \cap P)^{C} \in F$ and it is clear from the definition of $F$ that $(B \cup P)^{c} \cup\{p\}$ is also an element of $F$. But, this says that $\left|(B \cap P)^{C} u\{p\}\right| \cap(B \cap P)=\{p\}$. This implies that $\{p\} \in T^{*}$, which contradicts the assumption that $T^{*}$ is perfect. Therefore, for each $y \in U_{x}$ and for each $T^{\prime}$-open set $U$ containing $y$, there exists some $\alpha \in A$, such that $U \cap U_{x} \neq \emptyset$.

LEMMA 4. If $P \in T^{*}$ and $P$ has property $B$, then $P \in T^{\prime}$.

Proof. Let $x \in P$ and let $U_{x} \subset P$ be a $T^{*}$-open set such that $U_{x}=\left\{\begin{array}{cc}U & K_{\alpha}\end{array}\right\} \cup L$, where the $K_{\alpha}^{\prime}$ s and $L$ satisfy conditions (a) through (c) of Definition 3. We will show that for every $y \in U_{x}$, there exists a $T^{\prime}$ open set $U$, containing $y$, such that $U \subset U_{x}$.

For each $y \in L$, put $U(y)=\left\{\begin{array}{cc}U & U_{\alpha}\end{array}\right\} \cup\{y\}$. It follows from Lenma 3, that for each $y \in L, U\{y\}$ is an $N$-set since for every $p \in U(y)$ and every $T$-open set $U$ containing $p, U \cap U_{\alpha} \neq \emptyset$ for some $\alpha$.

We will show that $U(y) \in M$. Suppose not. Then, since $M$ is a maximal $N$-family, there must exist some $M \in M$, such that $M \cap U(y)$ is not an $N$-set. This implies there exists some $T$-open set $V$ containing $y$ such that $V \cap(M \cap U(y))$ does not contain a T-open set. It follows from the definition of an $N$-set, and the fact that $\{U(y)-\{y\}\}$ is $T$-open that $\{U(y)-\{y\}\} \cap M \cap V=\emptyset$. Therefore, $V \cap\left(M \cap U_{x}\right) \subset L$ since $U_{x}=\left\{U_{\alpha \in A}^{U} U_{\alpha}\right\} \cup L$. Put $\quad B=V \cap\left(M \cap U_{x}\right)$, then $B \in T^{*}$. Since $L$ is nowhere dense in $(X, T), \quad B$ must also be nowhere dense in $(X, T)$. Therefore, $H^{c} \in F$, since $F$ contains each set whose complement is nowhere dense in $(X, T)$. This implies that $\left(H^{c} \cap(y\}\right) \cap H=\{y\} \in T^{*}$, which contradicts the assumption that $T^{*}$ is perfect. Therefore, $U(y) \cap M$ must be an element of $M$, for all $M \in M$, and, so, $U(y) \in M$. 
This implies that $U(y) \in T^{\prime}$.

Since $\underset{\alpha \in A}{U} K_{\alpha}$ is $T$-dense in $\underset{\alpha \in A}{U} U_{\alpha}$, it follows from Lemma 2 that $\underset{\alpha \in A}{U} K_{\alpha}=F \cap\left(U_{\alpha \in A}^{U} U_{\alpha}\right)$, for some $F \in F$. Therefore, there exists an

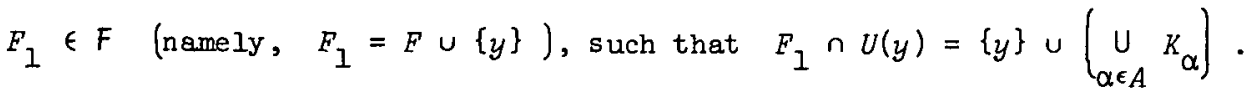
Since both $F_{1}$ and $U(y)$ are open in $T^{\prime}$, it follows that $U=\{y\} \cup\left(\bigcup_{\alpha \in A}^{U} K_{\alpha}\right)$ is open in $T^{\prime}$ and clearly, $U \subset U_{x}$. Therefore, for every $y \in U_{x}$, there exists a $T^{\prime}$-open set $U$ containing $y$ and contained in $U_{x}$. This implies that $U_{x}$ and, consequently $P$, is an element of $T^{\prime}$.

THEOREM 1. ( $\left.X, T^{\prime}\right)$ is maximal perfect.

Proof. Let $T^{*}$ be any perfect topology on $X$ such that $T^{*} \supset T^{\prime}$. Let $x \in X$ and let $U_{x}$ be any $T^{*}$-open set containing $x$. For each $y \in U_{x}$, let $K_{\alpha}$ be a subset of $U_{x}$, containing $y$, such that $K_{\alpha}$ is T-dense in some $U_{\alpha} \in T$ and $K_{\alpha} \subset U_{\alpha}$, if such a $K_{\alpha}$ exists. Otherwise, let $L=\left\{y \in U_{x} \mid\right.$ no such $K_{\alpha}$ exists $\}$. Clearly $U_{x}=\left\{\begin{array}{c}U K_{\alpha} \\ U_{\alpha}\end{array}\right\} U$, and, therefore, by Lemma $4, U_{x} \in T^{\prime}$ and $T^{\prime}$ is maximal perfect.

In the preceding, we constructed a maximal perfect topology $T^{*}$ on a perfect topological space $(X, T)$ by adding to $T$ a particular maximal filter $F$ of dense subsets of $(X, T)$ and a maximal $N$-family $M$. We will now show that if $\sigma$ is any perfect topology on $X$, larger than $T \vee F$, then $\sigma$ can be generated by $T, F$ and a family of $N$-sets. On the other hand, if $\gamma$ is a perfect topology, larger than $T \vee M$, we will give an example to show that $\gamma$ may not be generated by $T, M$ and a family of dense subsets of $(X, T)$.

Let $(X, T)$ be a perfect topological space and let $F$ be a maximal family of dense subset of $(X, T)$, such that, if $H$ is a nowhere dense subset of $(X, T)$, then $H^{c} \in F$. 
THEOREM 2. Let $\sigma$ be ony perfect topology on $X$ such that $\sigma \subset T \vee F$. If $G \in \sigma$ and $G \neq T \vee F$, then $G=M \cap F$, where $M$ is an $N$-set and $F \in F$.

Before presenting the proof of Theorem 2, we present a lemma which will be helpful in completing the proof of this theorem. The symbols used in Lemma 5 have the same meaning as in Theorem 2.

LEMMA 5. If $V$ is any $T$-open subset of $X$ arid $G \cap V \neq \emptyset$, then $G \cap V$ is T-dense in some T-open subset of $V$.

Proof. Suppose $G \cap V$ is not $T$-dense in any $T$-open subset of $V$. Then, $G \cap V$ is nowhere dense in $(X, T)$. This implies that $(G \cap V)^{c} \in F$. Let $y \in G \cap V$. Then $(G \cap V)^{c} \cup\{y\} \in F$ and since $\{y\}=\left[(G \cap V)^{c} v\{y\}\right] \cap(G \cap V)$, this implies that $\{y\} \in \sigma$, and contradicts the fact that $(X, \sigma)$ is perfect. Therefore, $G \cap V$ is $T$-dense in some $T$-open subset of $V$.

Proof of Theorem 2. Put $G=\left\{\underset{\alpha A}{U} K_{\alpha}\right\} \cup L$, where for each $\alpha \in A$, $K_{\alpha} \subset U_{\alpha}, U_{\alpha}$ is $T$-open and $K_{\alpha}$ is $T$-dense in $U_{\alpha}$; and no subset of $L$ is T-dense in any T-open set. Put $M=\left\{\bigcup_{\alpha A} U_{\alpha}\right\} \cup L$. We will show that $M$ is an $N$-set. Let $x \in M$. If $x \in U_{\alpha}$ for some $\alpha$, then clearly, for every $T$-open set $U$ containing $x, U \cap U_{\alpha}$ is a $T$-open set. If $x \in L$ and $U$ is a T-open set containing $x$, then it follows from Lemma 5, that $U \cap U_{\alpha} \neq \emptyset$, for some $\alpha$, so, again, $U \cap U_{\alpha}$ is a T-open set and is contained in $M$. Therefore, $M$ is an $N$-set.

Finally, we will show that for some $F \in F, F \cap M=G$. It follows from Lemma 2 that there exists some $F_{1} \in F$, such that $F_{1} \cap\left\{U_{\alpha} U_{\alpha}\right\}=G \cap\left\{\begin{array}{ll}U & U_{\alpha}\end{array}\right\}$. Put $F=F_{1} \cup\{x \mid x \in L\}$. Then $F \cap M=G$ and the theorem is established.

We now give an example of a maximal $I /-f a m i l y$ and a perfect topology $\gamma$ on $X$, such that $\gamma$ is larger than $T \vee M$ but $\gamma$ cannot be generated by $T, M$ and a family of dense subsets of $(X, T)$. 
EXAMPLE. Let $X$ be the closed unit interval and let $T$ be the usual topology on $X$. Let $K$ be the cantor subset of $X$ and let $c_{n}=\left(a_{n}, b_{n}\right)$, for $n=1,2, \ldots$, be the open intervals which are removed to form the Cantor set. Put $R_{n}=\left(0, a_{n}\right]$, and $G_{n}=\left[b_{n}, 1\right)$, for $n=1,2, \ldots$. Then for each $n, R_{n}$ and $G_{n}$ is an $N$-set. Let $M$ be a maximal $N$-Pamily which contains $R_{n}$ and $G_{n}$, for all $n$. Put $\gamma=(T \vee M) \vee K$. Then, $\gamma$ is perfect and $K$ is a $\gamma$-open set. It is easily seen that $K \neq M \cap F$, where $F$ is $T$-dense in $X$ and $M \in M$.

\section{References}

[1] I van Baggs, "A connected Hausdorff space which is not contained in a maximal connected space", (to appear).

[2] J. Pelham Thomas, "Maximal connected topologies", J. Austral. Math. Soc. 8 (1968), 700-705.

St Francis Xavier University,

Antigonish,

Nova Scotía,

Canada. 